\title{
ELABORAÇÃO DE IOGURTE GREGO DE LEITE DE BÚFALA E INFLUÊNCIA DA ADIÇÃO DE CALDA DE GINJA (Eugenia uniflora L.) NO TEOR DE ÁCIDO ASCÓRBICO E ANTOCIANINAS DO PRODUTO
}

\section{Preparation of greek yogurt of buffalo milk and influence of addition of sour cherry syrup (Eugenia uniflora L.) on the content of ascorbic acid and anthocyanins}

\author{
Elivaldo Nunes Modesto Junior ${ }^{*}$, Suane da Silva Soares ${ }^{2}$, \\ Deyse Dayan Freitas de Sousa ${ }^{2}$, Juliana Rodrigues do Carmo ${ }^{I}$, \\ Rogério Migdon Vieira da Silva ${ }^{3}$, Carmelita de Fátima Amaral Ribeiro ${ }^{2}$
}

\section{RESUMO}

A ginja (Eugenia uniflora L.) apresenta compostos fenólicos com ação antioxidante, ação hipoglicemiante e antirreumática, utilizada em distúrbios estomacais e como anti-hipertensivos. O leite de búfala exibe excelente qualidade nutricional destacando-se como principais características o elevado teor de proteínas com $25,55 \%$, tendo mais aminoácidos essenciais que o leite de vaca. $\mathrm{O}$ objetivo deste estudo foi elaborar um iogurte grego de leite de búfala com calda de ginja e avaliar a influência da adição de diferentes percentuais de calda $(10,20$ e $30 \% \mathrm{p} / \mathrm{p}$ ) sobre o teor de ácido ascórbico e antocianinas no produto elaborado. Os frutos foram coletados no município de Salvaterra e levados para o laboratório onde foram sanitizados e despolpados para utilização no iogurte. Os iogurtes foram elaborados com leite bubalino, açúcar e leite em pó integral. Os frutos de ginja, o leite de búfala e o iogurte elaborado foram avaliados quanto as suas características físico-químicas, microbiológicas e sensoriais. Os frutos apresentaram uma ótima relação sólidos totais tituláveis (SST)/acidez total titulável (ATT) e apresentaram teores de ácido ascórbico e antocianinas elevados $(124,08 \mathrm{mg} / 100 \mathrm{~g}$ e 179,27 m

1 Universidade Federal do Pará (UFPA), Av. Augusto Corrêa, 8856, 66075-970, Belém, PA, Brasil.Email: modesto.ufpa@outlook.com

2 Universidade do Estado do Pará (UEPA), Salvaterra, Marajó, PA, Brasil.

3 Laboratório Nacional Agropecuário no Pará, Belém, PA, Brasil.

* Autor para correspondência. 
Eqg Cianidina-3-glicosideo/100g respectivamente). O leite de búfala utilizado apresentou características físico-químicas de acordo com os padrões microbiológicos exigidos por lei. O aumento do percentual de calda de ginja no iogurte grego de búfala elevou significativamente os teores de ácido ascórbico e antocianinas no produto, demonstrando a possibilidade da produção de novos produtos com características nutricionais agregadas para inserção em novos mercados. O produto também apresentou da boa aceitação sensorial, sendo que as formulações com 10\%, 20\% e $30 \%$ de calda apresentaram percentuais de $87 \%$, $89 \%$ e $89 \%$ de aceitação pelos provadores respectivamente.

Palavras-chave: derivado lácteo; fruto regional; aspectos nutricionais; alimento alternativo.

\section{ABSTRACT}

The sour cherry (Eugenia uniflora L.) has phenolic compounds with antioxidant, hypoglycemic and anti-rheumatic actions, it used in stomach disorders and as antihypertensive agent. Buffalo milk exhibits excellent nutritional quality, with a high protein content of $25.55 \%$ as its main characteristic, with more essential amino acids than cow milk. The aim of this study was to develop a greek yogurt with buffalo milk with addition of distinct concentrations of sour cherry syrup (10, 20 and $30 \%$ $\mathrm{w} / \mathrm{w}$ ), on the content of ascorbic acid and anthocyanins in the final product. The fruits were collected in the municipality of Salvaterra and taken to the laboratory where they were sanitized and washed for use in yogurt. The yogurts were obtained with buffalo milk, sugar and milk powder. Sour cherry fruits, buffalo milk and yogurt prepared were assessed their physicochemical, microbiological and sensory characteristics. Fruits had a great ratio of total soluble solids/total titratable acidity and found high levels of ascorbic acid and anthocyanins $124.08 \mathrm{mg} / 100 \mathrm{~g}$ e 179.27 $\mathrm{m}$ Eqg Cyanidin-3-glycoside/100g respectively, and buffalo milk had good physicalchemical characteristics and it was within the microbiological standards. The increase in concentration of sour cherry syrup in the buffalo greek yogurt elevated significantly contents of ascorbic acid and anthocyanins, demonstrating the feasibility of producing new products with aggregate nutritional characteristics for insertion into new markets, in addition to good acceptance being the formulations with 10 , 20 e $30 \%$ with 87,89 e $89 \%$ of acceptance by tasters. food.

Keywords: dairy derivative; regional fruit; nutritional aspects; alternative

\section{INTRODUÇÃO}

A pitanga (Eugenia uniflora L.), conhecida em alguns lugares como ginja, é originária da região que se estende desde o Brasil Central até o Norte da Argentina. No entanto, por ser espécie de fácil adaptação, sua distri- buição se fez ao longo de quase todo o território brasileiro, bem como em várias partes do mundo (SANCHOTENE, 1989; BEZERRA et al., 2000). Este fruto apresenta substâncias fenólicas com ação antioxidante, ação hipoglicemiante e antirreumática que também são utilizadas em distúrbios estomacais 
e como anti-hipertensiva (ASSUMPÇÃO; NASCIMENTO, 2000; AURICHIO; BACCHI, 2003). No município de Salvaterra, localizado na região Norte do Brasil, o fruto é bastante consumido pela população local, sendo encontrado principalmente nos quintais dos moradores locais e até mesmo em vias públicas.

O leite de búfala exibe excelente qualidade nutricional destacando-se como principais características o elevado teor de proteínas $(25,55 \%)$ e mais aminoácidos essenciais que o leite de vaca (VERRUMA; SALGADO, 1994), alto teor gordura apresentando maior valor calórico que o leite bovino e a alta porcentagem de minerais, sendo o mais importante o cálcio. A utilização do leite de búfala para a produção industrial de iogurte tem mostrado que o produto obtido apresenta características sensoriais, nutricionais e físico-químicas diferentes do produto produzido exclusivamente de leite bovino (AHMAD et al., 2008; BORGES et al., 2009; CUNHA NETO et al., 2005).

O iogurte de leite de búfala apresenta uma textura firme, sem necessitar de fortalecimento com extrato seco desengordurado (ESD), o que representa a obtenção de um derivado com maior rendimento econômico, repercutindo assim na consistência e no sabor (BORGES et al., 2009).

Diante disso, o objetivo deste estudo foi elaborar um iogurte grego de leite de búfala, avaliar a influência da adição de diferentes concentrações de calda de ginja no mesmo e quantificar o teor de ácido ascórbico e antocianinas provenientes da adição da calda no produto elaborado.

\section{MATERIAL E MÉTODOS}

O presente estudo foi desenvolvido no laboratório de Tecnologia de Alimentos da Universidade do Estado do Pará, Salvaterra, Marajó, Pará.

\section{Obtenção da matéria-prima}

Os frutos foram coletados no município de Salvaterra e acondicionados em caixas plásticas para evitar injurias mecânicas. Os mesmos foram transportados até o laboratório de Tecnologia de Alimentos onde passaram por um processo de seleção. Os frutos que apresentaram lesões foram separados dos sadios e passaram por sanitização em água clorada a $10 \mathrm{ppm}$ por $10 \mathrm{~min}$.

\section{Matérias-primas para produção do iogurte}

As matérias-primas utilizadas para a elaboração do iogurte grego de leite de búfala adicionado de calda de polpa de ginja foram: leite de búfala in natura; polpa de ginja; leite integral UHT - CCGL ${ }^{\circledR}$; açúcar refinado - UNIÃO ${ }^{\circledR}$; cultura láctea liofilizada - BioRich ${ }^{\circledR}$ utilizada para o processo de fermentação composta por bactérias Lactobacillus acidophilus e Streptococcus subsp. thermophilus.

\section{Elaboração de calda a partir de polpa de fruto ginja (Eugenia Uniflora L.)}

A elaboração das caldas a partir da polpa foi realizada de acordo com as concentrações de $10 \%$, 20\% e 30\% para as massas de iogurte e foram utilizados: açúcar, polpa de fruta e água de acordo com as concentrações da Tabela 1.

Para a produção das caldas a polpa, o açúcar e água foram homogeneizados e levados para cocção a $80{ }^{\circ} \mathrm{C} / 15$ minutos, tempo suficiente para que a calda adquirisse a consistência necessária para ser incorporada nos exemplares de iogurtes.

Elaboração do iogurte grego de leite de búfala com calda de ginja

Para a produção do iogurte grego de 
leite de búfala foram utilizadas as seguintes concentrações (Tabela 2).

Tabela 2 - Concentrações de matérias-primas para produção de iogurte grego de leite de búfala

\begin{tabular}{cc}
\hline Matéria-prima & Valor percentual (\%) \\
\hline Leite bubalino & 94,33 \\
Açúcar & 3,30 \\
Leite em pó integral & 2,37 \\
\hline
\end{tabular}

O leite de búfala in natura foi pasteurizado em recipiente previamente higienizado para evitar possíveis contaminações. O leite foi aquecido a $90{ }^{\circ} \mathrm{C}$ durante 3 minutos, já adicionado do leite em pó e açúcar previamente pesados. Em seguida, foi resfriado até a temperatura de $42{ }^{\circ} \mathrm{C}$ para a inoculação da cultura láctica, sendo 40 gramas para cada 1 litro de leite, de acordo com o fabricante $\left(\right.$ BioRich $\left.^{\circledR}\right)$. Após a inoculação, o leite foi levado para incubação em BOD (marca $\mathrm{BIOFOCO}^{\circledR}$ ) até atingir o $\mathrm{pH}$ de 4,6.

No término do processo de fermentação, o recipiente com iogurte passou por um processo resfriamento a fim de se inativar o processo fermentativo dos microrganismos produtores de ácido lático. Em seguida, a massa de iogurte foi transferida para sacos de algodão devidamente esterilizados para o processo de dessoramento a $10{ }^{\circ} \mathrm{C}$ por 5 horas. Após a dessoragem, a massa de iogurte foi pesada e dividida de acordo com as formulações definidas e então adicionadas das formulações de calda de polpa do fruto ginja sendo homogeneizadas em batedeira (marca $\mathrm{ARNO}^{\circledR}$ ).

\section{Análises físico-químicas}

Para a caracterização físico-química dos frutos de ginja e dos iogurtes gregos de leite bubalino e ginja foram realizadas as seguintes análises: pH (IAL, 2005), acidez total titulável (IAL, 2005), umidade (AOAC, 2000), cinzas (IAL, 2005), sólidos solúveis (IAL, 2005), açúcares redutores (MILLER, 1959), lipídeos (AOAC, 1995), densidade (IAL, 2005), ácido ascórbico (SILVA et al., 1995) e antocianinas totais (ASKAR; TREPTOW, 1993).

\section{Análise microbiológica}

Foram realizadas pesquisas de Coliformes a $35{ }^{\circ} \mathrm{C}$, Coliformes a $45^{\circ} \mathrm{C}$ por meio de plaqueamento em placas de Petrifilm $3 \mathrm{M}^{\mathrm{TM}}$ e Staphylococcus aureus (AOAC, 2003) no leite bubalino e nas formulações de iogurte de acordo com Brasil (2007).

\section{Análise Sensorial}

Participaram da análise 50 provadores não treinados que avaliaram o produto através de uma escala hedônica de 9 pontos, onde se atribuiu valores de 1 (desgostei muitíssimo) a 9 (gostei muitíssimo) para os atributos sensoriais sendo eles a aparência, aroma, sabor, consistência, impressão global, conforme descrito por Dutcosky (2007). A aceitação

Tabela 1 - Formulações para a elaboração das caldas de ginja para enriquecimento do iogurte grego

\begin{tabular}{cccc}
\hline \multirow{2}{*}{ Matérias-primas } & \multicolumn{3}{c}{ Formulações de caldas } \\
\cline { 2 - 4 } & $10 \%$ & $20 \%$ & $30 \%$ \\
\hline Polpa de ginja (\%) & 50,06 & 51,77 & 53,60 \\
Açúcar (\%) & 33,01 & 35,29 & 37,43 \\
Água (\%) & 13,39 & 12,94 & 12,51 \\
\hline
\end{tabular}


foi avaliada através do cálculo de índice de aceitabilidade (IA), calculado a partir da expressão: IA $(\%)=\mathrm{A} \times 100 / \mathrm{B}$, onde $\mathrm{A}=$ nota média obtida para o produto, e $\mathrm{B}=$ nota máxima dada ao produto.

\section{Análise estatística}

Os resultados das análises realizadas foram avaliados através da análise de variância (ANOVA) e teste de Tukey a 5\% de probabilidade nos programas estatísticos Assistat 7.1 e Statistica 7. A análise multivariada qualitativa (Análise de Componentes Principais ACP) também foi utilizada na avaliação dos dados das análises, com o emprego do programa Statistica 7.

\section{RESULTADOS E DISCUSSÃO}

\section{Caracterização físico-química do fruto de ginja}

Os resultados das análises físico-químicas do fruto de ginja no estádio maduro podem ser observados na Tabela 3 .

$\mathrm{O}$ valor encontrado para o $\mathrm{pH}$ do fruto foi de 3,53, classificando-o como ácido, sendo este valor próximo ao encontrado por Bagetti (2009) $(3,38)$ em frutos de pitanga. O $\mathrm{pH}$ encontrou-se fora do estabelecido pela legislação para polpa do fruto ginja (BRASIL, 2000).

A acidez titulável em mEq de $\mathrm{NaOH} /$
$100 \mathrm{~g}$ foi de $0,04 \%$ no fruto estudado, sendo inferior aos valores encontrados por Barreto (2011) (1,62\%) e Bagetti (2009) que foi de $0,67 \%$ em seus estudos com ginja.

Para o mercado consumidor de frutas frescas e/ou processadas, a relação sólidos totais tituláveis (STT)/acidez total titulável (ATT) elevada é desejável (AGUIAR, 2006), pois a relação da acidez do fruto com sua doçura está ligada diretamente com o estádio de maturação. O valor da relação para esse estudo foi de 275 , indicando doçura adequada.

O teor de ácido ascórbico no fruto maduro e foi de $124,08 \mathrm{mg} / 100 \mathrm{~g}$. Santos et al. (2003) encontraram para pitanga vermelha $33,00 \mathrm{mg} / 100 \mathrm{~g}$ e $38,35 \mathrm{mg} / 100 \mathrm{~g}$ para pitanga roxa. Tais valores foram inferiores aos do presente estudo. Valores inferiores também foram encontrados por Oliveira, Figuêiredo e Queiroz (2006) (13,42 mg/100g) em frutos de pitanga provenientes da região de Campina Grande, Paraíba.

$\mathrm{O}$ teor de antocianinas para o fruto de ginja maduro foi de $124,076 \mathrm{mg}$ de cianidina-3-glicosídeo/100g. Lima, Mélo e Lima (2005) encontraram valores inferiores ao do presente estudo para frutos de pitanga de estádio roxo $(16,23 \mathrm{mg} / 100 \mathrm{~g})$.

\section{Caracterização físico-química e mi- crobiológica do leite}

Na Tabela 4 estão apresentados os re-

Tabela 3 - Valores médios para a caracterização físico-química do fruto no estádio maduro

\begin{tabular}{lcc}
\hline \multicolumn{1}{c}{ Parâmetros } & \multicolumn{2}{c}{ Estádios } \\
\cline { 2 - 3 } & Maduro & BRASIL (2000) \\
\hline $\mathrm{pH}$ & $3,53 \pm<0,01$ & 2,5 a 3,4 \\
Acidez titulável (mEq de NaOH/100g) & $0,04 \pm<0,01$ & Mín. 0,92 \\
Relação SST/ATT & 275 & - \\
Antocininas (mEqgCianidina-3-glicosideo/100g) & $179,27 \pm 1,80$ & - \\
Ácido Ascórbico (mg/100g) & $124,08 \pm 0,23$ & - \\
\hline
\end{tabular}


sultados obtidos para a caracterização físicoquímica do leite bubalino.

Devido à ausência de legislação específica para o leite bubalino os resultados foram comparados de acordo com os descritos pela SAA (Secretaria de Agricultura e Abastecimento de São Paulo) e com os parâmetros para leite bovino. O valor de $\mathrm{pH}$ para o leite bubalino $(6,85)$ apresentou-se de acordo com o estabelecido pela SAA. Valores superiores de $\mathrm{pH}$ foram encontrados por Borges et al. (2009) $(6,65)$ e Guerra et al. (2005) $(6,80)$ para o mesmo tipo de leite.

O teor de umidade do leite bubalino $(83,2 \%)$ diferiu em relação ao leite bovino $(86,50 \%)$. Estas variações, no entanto, se dão devido à diferença entre as raças, pois o leite bubalino apresenta um maior teor de sólidos. Oliveira et al. (2009) encontraram em seus estudos com leite de bubalino valor de $20,1 \%$ de sólidos totais.

O valor de acidez do leite bubalino $(0,12 \%)$ foi inferior ao estabelecido pela SAA $(0,14 \%$ a $0,23 \%)$ e legislação bovina $(0,78 \%$ a 1,21\%) em ácido láctico.

O teor de gordura encontrado para o leite bubalino $(9,0 \%)$ mostrou-se de acordo com os padrões legais estipulados pela secretaria de abastecimento do estado de São Paulo. Tal valor foi superior aos encontrados por Cunha Neto et al. (2005) (6,82\%), Oliveira et al. (2009) (8,6\%) e Coelho et al. (2004) $(6,8 \%)$ para leite bubalino.

A densidade do leite bubalino (1.056, $58 \mathrm{~g} / \mathrm{L})$ foi semelhante ao valor encontrado por Cunha Neto et al. (2005) (1.033,84 g/L) em seus estudos com leite bubalino.

O teor de cinzas do leite bubalino $(0,5 \%)$ foi de acordo com o estabelecido pela SAA e inferior aos limites para leite bovino. Guerra et al. (2005) encontraram valor de 4,0\% para cinzas.

O teor de sólidos totais do leite bubalino $(16,8 \%)$ foi semelhante aos estudos de Lopes et al. (2009) (16,8\%) e Coelho et al. (2004) (17,2\%).

$\mathrm{Na}$ Tabela 5 estão apresentados os resultados para as análises microbiológicas realizadas no leite bubalino in natura.

De acordo com os resultados obtidos para o leite in natura, a legislação vigente (BRASIL, 2002) permite contagem em UFC/g de até $1 \times 10^{4}$ colônias e pode-se verificar que a contagem de Staphylococcus aureus apresentou contagens acima do permitido, indicando alguma falha durante o processo de manipulação desse leite. No entanto, os valores de coliformes, que são indicadores de qualidade, mostraram que o leite foi manipulado com boas práticas.

Tabela 4 - Caracterização físico-química do leite bubalino

\begin{tabular}{cccc}
\hline Parâmetros & Leite & *Parâmetro (SAA) & $\begin{array}{c}\text { **Parâmetro } \\
\text { (Leite bovino) }\end{array}$ \\
\hline $\mathrm{pH}$ & $6,85 \pm 0,08$ & 6,40 a 6,80 & - \\
Umidade (\%) & $83,2 \pm 0,57$ & - & 86,50 \\
Acidez (\% ácido lático) & $0,13 \pm<0,01$ & $0,14 \%$ a 0,23 & 0,78 a 1,21 \\
Lipídeos (\%) & $9,00 \pm 0,05$ & Mínimo 4,5 & Mínimo 3,0 \\
Densidade (g/L) & $1.056,58 \pm 0,08$ & 1,028 a 1,034 & 1,028 a 1,034 \\
Cinzas (\%) & $0,5 \pm<0,01$ & Máximo 8,4 & 0,73 \\
Sólidos Totais $(\%)$ & $16,8 \pm 0,03$ & - & - \\
\hline
\end{tabular}

*Parâmetros do Sistema de Agricultura e Abastecimento de São Paulo; ** BRASIL (2011). 


\section{Caracterização físico-química de iogurte grego sabor ginja}

Na Tabela 6 estão apresentados os resultados para a caracterização físico-química das formulações de iogurte grego com diferentes concentrações de calda de ginja.

No que diz respeito ao $\mathrm{pH}$ do iogurte é possível verificar que as formulações apresentaram diferença significativa entre si exceto as formulações com $20 \%$ e $30 \%$ de calda de ginja ( $p>0,05)$. Neves (2007) encontrou valores de $\mathrm{pH}$ de 4,6 para iogurte de açaí, valores superiores ao encontrado nesse estudo.

O percentual de acidez das amostras, SA (sem adição de calda) e 10\% encontraram-se em desacordo ao estabelecido pela legislação para leite fermentado (BRASIL, 2007). Apenas as formulações de $20 \%$ e de $30 \%$ de calda de ginja, apresentaram-se de acordo com a legislação que prevê valores entre $0,6 \%$ e $2 \%$ de ácido lático (BRASIL, 2007).

O teor de umidade das formulações de $20 \%$ e $30 \%$ não diferiram entre si $(\mathrm{p}>0,05)$ assim como para os valores de $\mathrm{pH}$. Já para o teor de sólidos solúveis apenas a formulação SA apresentou diferença significativa $(\mathrm{p}<0,05)$ em relação às demais com valor de $15{ }^{\circ}$ Brix. As formulações $10 \%, 20 \%$ e $30 \%$ apresentaram teor de sólidos solúveis de $24{ }^{\circ}$ Brix.

Os teores de lipídios mostraram-se de acordo com a legislação para leite fermentado que preconiza padrões de no mínimo 3\% de lipídios (BRASIL, 2007).

Tabela 5 - Resultado das análises microbiológicas para o leite de búfala in natura

\begin{tabular}{cccc}
\hline & \multicolumn{3}{c}{ Análises } \\
\cline { 2 - 4 } & $\begin{array}{c}\text { Coliformes a } 35^{\circ} \mathrm{C} \\
(\mathrm{UFC} / \mathrm{g})\end{array}$ & $\begin{array}{c}\text { Coliformes a } 45^{\circ} \mathrm{C} \\
(\mathrm{UFC} / \mathrm{g})\end{array}$ & $\begin{array}{c}\text { Staphylococcus aureus } \\
(\mathrm{UFC} / \mathrm{g})\end{array}$ \\
\hline $\begin{array}{c}\text { Leite } \text { in natura } \\
* \text { Padrões }\end{array}$ & $3,0 \times 10^{3}$ & $<3$ & $1,5 \times 10^{4}$ \\
\hline
\end{tabular}

*BRASIL, 2002.

Tabela 6 - Caracterização físico-química de iogurte grego com diferentes concentrações de calda de ginja

\begin{tabular}{ccccc}
\hline \multirow{2}{*}{ Parâmetros } & \multicolumn{4}{c}{ Formulações } \\
\cline { 2 - 5 } & $* * *$ SA & $10 \%$ & $20 \%$ & $30 \%$ \\
\hline pH & $4,13^{\mathrm{a}} \pm 0,05$ & $3,90^{\mathrm{b}} \pm 0,00$ & $3,63^{\mathrm{c}} \pm 0,05$ & $3,63^{\mathrm{c}} \pm 0,05$ \\
*Acidez (\%) & $0,39^{\mathrm{c}} \pm 0,01$ & $0,39^{\mathrm{c}} \pm 0,01$ & $0,63^{\mathrm{a}} \pm 0,00$ & $0,59^{\mathrm{b}} \pm 0,00$ \\
Umidade (\%) & $68,17^{\mathrm{a}} \pm 0,04$ & $60,30^{\mathrm{b}} \pm 0,01$ & $58,07^{\mathrm{c}} \pm 0,00$ & $58,26^{\mathrm{c}} \pm 0,01$ \\
$* *$ SST $\left({ }^{\circ}\right.$ Brix) & $15,00^{\mathrm{b}} \pm 0,00$ & $24,00^{\mathrm{a}} \pm 0,00$ & $24,00^{\mathrm{a}} \pm 0,00$ & $24,00^{\mathrm{a}} \pm 0,00$ \\
Lipídios $(\%)$ & $42,49^{\mathrm{a}} \pm 0,01$ & $39,85^{\mathrm{b}} \pm 0,11$ & $19,88^{\mathrm{d}} \pm 0,63$ & $23,37^{\mathrm{c}} \pm 0,21$ \\
$* * *$ AR (\%) & $4,70^{\mathrm{b}} \pm 0,90$ & $4,8^{\mathrm{b}} \pm 0,60$ & $4,90^{\mathrm{ab}} \pm 0,12$ & $6,90^{\mathrm{a}} \pm 1,01$ \\
Cinzas $(\%)$ & $0,50^{\mathrm{a}} \pm 0,02$ & $0,70^{\mathrm{a}} \pm 0,03$ & $1,20^{\mathrm{a}} \pm 0,22$ & $0,50^{\mathrm{a}} \pm 0,03$ \\
\hline
\end{tabular}

Letras iguais na mesma linha não divergem significativamente no teste de Tukey $(\mathrm{p}>0,05)$, *Acidez $(\%$ de ácido lático); ** SST - Sólidos solúveis totais; ***SA - Sem adição de calda; *** AR - Açúcares Redutores. 
O valor de açúcares redutores das formulações SA, $10 \%$ e $20 \%$ não diferiram estatisticamente $(\mathrm{p}>0,05)$ com valores de $4,7 \%$, $4,8 \%$ e $4,9 \%$, respectivamente. Borges et al. (2009) encontraram em seus estudos valor de AR de $9,47 \%$ para iogurte produzido a partir de leite de búfala e para iogurte bovino de $7,27 \%$, sendo esses valores superiores ao do iogurte grego sabor ginja elaborado nesse estudo.

O teor de cinzas não apresentou diferenças significativas $(\mathrm{p}>0,05)$ entre as formulações apresentando uma média de $0,7 \%$. Medeiros et al. (2007) ao analisarem iogurtes de marca comercial, encontraram $0,46 \%$ de cinzas, valor inferior à média desse estudo. Esse parâmetro não é contemplado pela legislação brasileira vigente (BRASIL, 2007) e é profundamente enunciado pela matériaprima, podendo ser consequência do elevado teor de cinzas do leite bubalino (VERRUMA et al., 1993).

Na Tabela 7 estão apresentados os resultados das análises de quantificação de ácido ascórbico (AA) e antocianinas nos iogurtes grego de leite de búfala com diferentes concentrações de calda de ginja.

De acordo com os resultados para o teor de ácido ascórbico (AA), apenas as formulações $10 \%$ e $20 \%$ não apresentaram diferen- ça significativa $(\mathrm{p}>0,05)$, contudo a formulação $30 \%$ apresentou $141,93 \mathrm{mg} / 100 \mathrm{~g}$, indicando um aumento de $453 \%$ em relação a formulação SA $(25,66 \mathrm{mg} / 100 \mathrm{~g})$. Souza (2011), encontrou em seu estudo de elaboração de iogurte com polpas amazônicas uma variação de 140,22 mg/100g a 240,40 $\mathrm{mg} / 100 \mathrm{~g}$ de ácido ascórbico. Dados de vitamina $\mathrm{C}$ em derivados lácteos são escassos, mas Favaro-Trindade et al. (2006) relataram valores de $147,7 \mathrm{mg} / 100 \mathrm{~g}$ em sorvetes de acerola fermentado, valor próximo ao da formulação de $30 \%$ e superior aos demais. É possível verificar que todas as formulações apresentaram teor de vitamina $\mathrm{C}$ acima de $60 \mathrm{mg} / 100 \mathrm{~mL}$, valor de ingestão diária recomendada para pessoas adultas e saudáveis (ANVISA, 2005).

A concentração de antocianinas aumentou de forma significativa $(\mathrm{p}<0,05) \mathrm{com}$ o aumento da concentração de polpa de ginja nas caldas adicionadas com valores respectivos de 39,39 mg/100g, 49,80 mg/100g e $53,77 \mathrm{mg} / 100 \mathrm{~g}$. Costa et al. (2012) ao desenvolverem iogurte adicionado de polpa de juçaí, concluíram que devido a adição de polpa do fruto da palmeira Euterpe edulis Martius, que é rico em nutriente e antioxidantes, seu produto final também seria uma boa fonte de antocianinas e minerais

Tabela 7 - Resultados das análises de ácido ascórbico e antocianinas no iogurte grego de leite de búfala com diferentes concentrações de calda de ginja

\begin{tabular}{lcccc}
\hline \multirow{2}{*}{ Parâmetros } & $*$ FA & $10 \%$ & $20 \%$ & $30 \%$ \\
\cline { 2 - 5 } & $25,66^{\mathrm{c}} \pm 0,77$ & $113,38^{\mathrm{b}} \pm 0,85$ & $122,59^{\mathrm{b}} \pm 8,44$ & $141,93^{\mathrm{a}} \pm 0,75$ \\
\hline $\begin{array}{l}\text { Ác. Ascórbico } \\
(\mathrm{mg} / 100 \mathrm{~g})\end{array}$ & $* *$ nd & $39,40^{\mathrm{c}} \pm 0,28$ & $49,81^{\mathrm{b}} \pm 0,00$ & $53,77^{\mathrm{a}} \pm 0,05$ \\
\hline $\begin{array}{l}\text { Antocianinas } \\
\text { (mEqgCianidina- }\end{array}$ & & & & \\
3-glicosideo/100g) & & & & \\
\hline
\end{tabular}

Letras iguais na mesma linha não divergem significativamente no teste de Tukey $(\mathrm{p}>0,05)$. ${ }^{*} \mathrm{SA}-$ Sem adição de calda; **nd - não detectado. 
Na Figura 1 são mostrados os resultados gráficos (scores) que revelam a separação das formulações e os parâmetros físico-químicos que mais influenciam na separação.

O primeiro componente principal (CP 1) explicou $82,84 \%$ e o segundo (CP 2) explicou $14,67 \%$ da variância dos dados. De acordo com a ACP a maior parte dos parâmetros físico-químicos apresentou-se no $2^{\circ}$ quadrante (gráfico A) onde está localizada a formulação de 30\% (gráfico B), mostrando dessa forma, que essa formulação foi a que mais sofreu influência desses parâmetros, dentre eles os teores de ácido ascórbico e antocianinas.

\section{Caracterização microbiológica do iogurte grego}

Na Tabela 8 estão apresentados os resultados para as análises microbiológi-
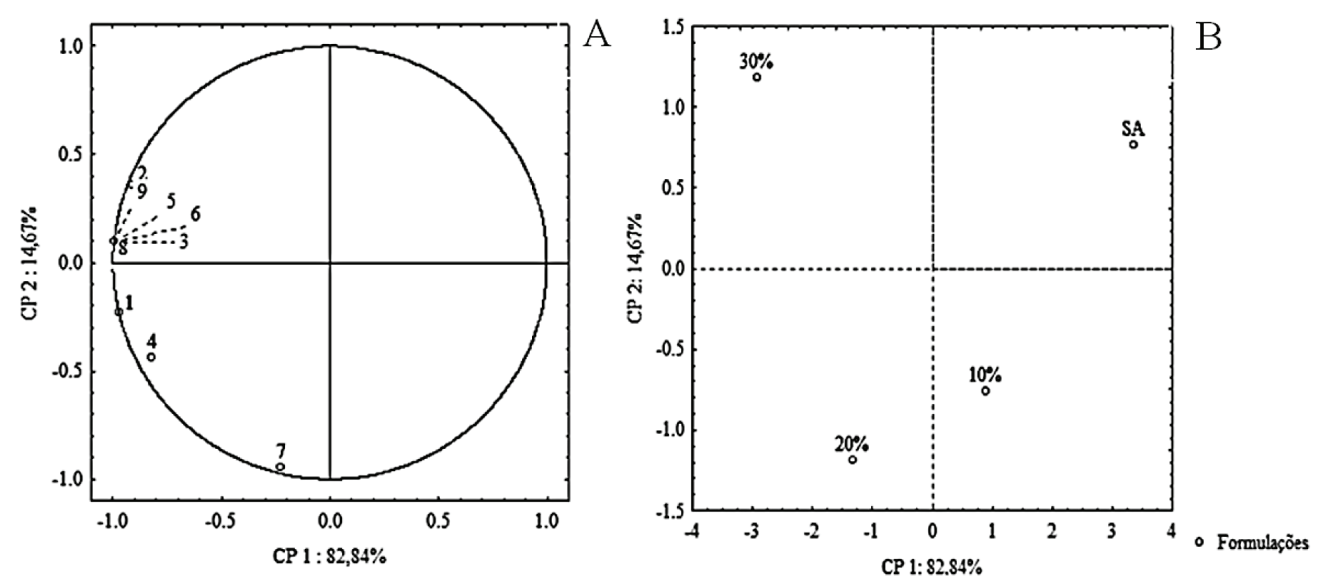

Figura 1 - Componentes principais (CP 1 e CP 2) dos dados obtidos na caracterização físicoquímica. (A) Gráfico de Scores para os parâmetros físico-químicos: 1 - pH; 2 - Acidez; 3 - Umidade; 4 - SST; 5 - Lipídios; 6 - AR; 7- Cinzas; 8 - Ác. Ascórbico; 9 - Antocianinas. (B) Gráfico de Scores para as formulações.

Tabela 8 - Resultado das análises microbiológicas para o iogurte grego de leite de búfala com diferentes concentrações de calda de ginja

\begin{tabular}{cccc}
\hline & \multicolumn{3}{c}{ Análises } \\
\cline { 2 - 4 } Formulações & $\begin{array}{c}\text { Coliformes a } 35^{\circ} \mathrm{C} \\
(\mathrm{UFC} / \mathrm{g})\end{array}$ & $\begin{array}{c}\text { Coliformes a } 45{ }^{\circ} \mathrm{C} \\
(\mathrm{UFC} / \mathrm{g})\end{array}$ & $\begin{array}{r}\text { Staphylococcus aureus } \\
(\mathrm{UFC} / \mathrm{g})\end{array}$ \\
\hline$* \mathrm{SA}$ & $\leq 3$ & $\leq 3$ & $\leq 3$ \\
$10 \%$ & $\leq 3$ & $\leq 3$ & $\leq 3$ \\
$20 \%$ & $\leq 3$ & $\leq 3$ & $\leq 3$ \\
$30 \%$ & $\leq 3$ & $\leq 3$ & $\leq 3$ \\
$* *$ Legislação & $10^{2} \mathrm{NMP} / \mathrm{mL}$ & $10 \mathrm{NMP} / \mathrm{mL}$ & - \\
\hline
\end{tabular}

*Sem adição de calda; ** IN nº 46 de 2007 - amostras indicativas (BRASIL, 2007). 
cas realizadas nos iogurtes com diferentes proporções de calda de polpa de ginja, analisaos quanto coliformes a $35^{\circ} \mathrm{C}, 45^{\circ} \mathrm{C}$ e Staphylococcus aureus.

De acordo com a Tabela 8 os resultados mostram a ausência de microrganismos nas formulações elaboradas, indicando valores inferiores aos preconizados pela legislação vigente. Logo, a partir desses resultados é possível indicar que os produtos estavam dentro dos padrões microbiológicos, demons- trando assim que o seu preparo foi feito seguindo as boas práticas de fabricação para produtos alimentícios.

\section{Avaliação sensorial}

Na Tabela 9 estão apresentados os valores médios para os resultados da análise sensorial quanto os atributos avaliados cor, aparência, aroma, consistência, sabor e impressão global.

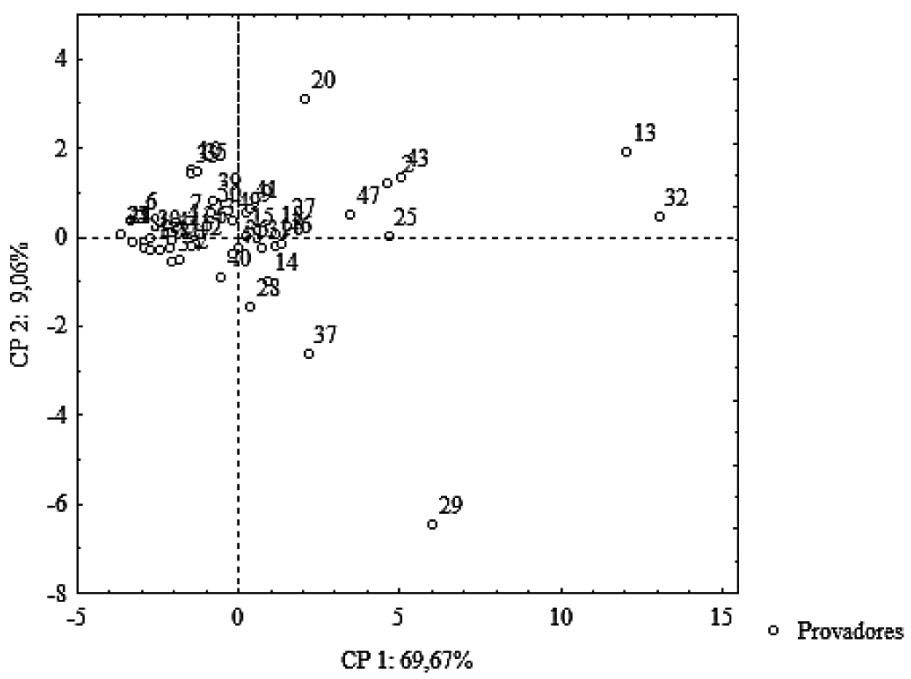

Figura 2 - Representação gráfica dos consumidores em relação aos atributos avaliados

Tabela 9 - Resultados das análises sensoriais dos iogurtes grego de leite de búfala com calda de ginja

\begin{tabular}{cccc}
\hline & \multicolumn{3}{c}{ Formulações } \\
\cline { 2 - 4 } Atributos & $10 \%$ & $20 \%$ & $30 \%$ \\
\hline Cor & $8,00^{\mathrm{a}} \pm 2,03$ & $8,00^{\mathrm{a}} \pm 1,83$ & $8,00^{\mathrm{a}} \pm 2,10$ \\
Aparência & $8,00^{\mathrm{a}} \pm 1,79$ & $8,00^{\mathrm{a}} \pm 1,76$ & $8,00^{\mathrm{a}} \pm 1,69$ \\
Aroma & $8,00^{\mathrm{a}} \pm 2,34$ & $7,00^{\mathrm{a}} \pm 2,25$ & $8,00^{\mathrm{a}} \pm 1,98$ \\
Consistência & $8,00^{\mathrm{a}} \pm 1,57$ & $8,00^{\mathrm{a}} \pm 1,41$ & $8,00^{\mathrm{a}} \pm 1,36$ \\
Sabor & $8,00^{\mathrm{a}} \pm 1,99$ & $8,00^{\mathrm{a}} \pm 1,73$ & $8,00^{\mathrm{a}} \pm 1,50$ \\
Impressão Global & $8,00^{\mathrm{a}} \pm 1,36$ & $8,00^{\mathrm{a}} \pm 1,18$ & $8,00^{\mathrm{a}} \pm 1,14$ \\
\hline
\end{tabular}

Letras diferentes, na mesma coluna, indicam diferenças significativas pelo teste de Tukey, $(\mathrm{p}<0,05)$. 
Observa-se na Tabela 9 que não houve diferença significativa $(\mathrm{p}>0,05)$ entre as formulações de iogurte grego para os atributos sensoriais, ambos apresentaram boas médias de aceitação de acordo com o teste da escala hedônica, fixando-se os valores em médias $(8,00)$, que indica "Gostei muito".

Os índices de aceitação dos iogurtes elaborados de leite de búfala com calda de ginja foram satisfatórios para as três formulações, sendo as de $10 \%, 20 \%$ e $30 \%$ com os índices respectivos de $87 \%$, 89\% e $89 \%$. Quanto às características sensoriais, para que o produto seja aceito, é necessário que o seu índice de aceitabilidade seja igual ou superior a 70\% (DUTCOSKY, 2007).

A Figura 2 apresenta o gráfico de scores para os atributos avaliados na análise sensorial. O primeiro componente principal (CP 1) explicou $69,67 \%$ e o segundo componente principal (CP 2), 9,06\% da variância dos dados.

Observou-se que os provadores estão localizados na região central do gráfico, não estando correlacionados com nenhum dos dois componentes. Portanto, os mesmos não discriminam as formulações elaboradas. Isso corrobora com o teste de aceitação (Tabela 9), onde não é observada diferença significativa $(p>0,05)$ entre as formulações.

\section{CONCLUSÕES}

O leite bubalino e os frutos de ginja apresentaram boas características físico-químicas, sendo que no fruto foram encontrados teores de ácido ascórbico e antocianinas elevados quando comparados aos estudos de outros autores sobre o mesmo fruto. O aumento da concentração de calda de ginja no iogurte grego de búfala elevou os teores de ácido ascórbico e antocianinas no produto, sendo que a formulação com $30 \%$ de calda de ginja apresentou valores elevados de ácido ascórbico e antocianinas, oriundos do amadurecimento do fruto, indicando possíveis propriedades bioativas e antioxidantes. O teor de ácido ascórbico encontrado no produto adicionado com as caldas foi superior ao recomendado para uma ingestão diária. Os resultados do índice de aceitabilidade e intenção de compra foram superiores a $85 \%$ para todas as formulações.

\section{REFERÊNCIAS}

AGÊNCIA NACIONAL DE VIGILÂNCIA SANITÁRIA (ANVISA). Resolução de Diretoria Colegiada, RDC n ${ }^{\circ} 269$, de 21 de outubro de 2005. Aprova o Regulamento Técnico sobre a ingestão diária recomendada (IDR) de proteína, vitaminas e minerais. Diário Oficial da República Federativa do Brasil, Brasília, 23 set. 2005.

AGUIAR, L. P. Qualidade e potencial de utilização de bacuris oriundos da região Meio-Norte. Fortaleza, 2006, 122f. Dissertação (Mestrado em Tecnologia de Alimentos) -Universidade Federal do Ceará.

AHMAD, S. et al. Effects of acidification on physic-chemical characteristics of buffalo milk: A comparison with cow's milk. Food chemistry, v. 106, p. 11-17, 2008.

ASKAR, A.; TREPTOW, H. Quality assurance in tropical fruit processing. New York: Springer-Verlag, 1993. 231p.

ASSOCIATION OF OFFICIAL ANALYTICAL CHEMISTS (AOAC). Official Methods of Analysis of AOAC International. $16^{\mathrm{a}}$ ed, Washington: AOAC international, 1995. $1141 \mathrm{p}$.

ASSOCIATION OF OFFICIAL ANALYTICAL CHEMISTS (AOAC). Official Methods of Analysis of AOAC International. $17^{\mathrm{a}} \mathrm{Ed}$, 2 rev, Gaithersburg: AOAC international, 2003.

ASSUMPÇÃO, J.; NASCIMENTO, M. T. Estrutura e composição florística de quatro 
formações vegetais de restinga no complexo lagunar Grussaí/Iquipari, São João da Barra, RJ, Brasil. Acta Botanica Brasilica, v. 14, n. 3, p. 301-315, 2000.

AURICCHIO, M. T.; ACCHI, E. M. Folhas de Eugenia uniflora L. (pitanga: propriedades farmacobotânicas, químicas e farmacológicas. Revista Instituto Adolfo Lutz, v. 62, n. 1, p. 55-61, 2003.

BAGETTI, M. Caracterização físico-química e capacidade antioxidante de pitanga (Eugenia uniflora L.). 2009. 84 f. Dissertação (Mestrado em Ciência e Tecnologia de Alimentos) - Universidade Federal de Santa Maria, Santa Maria, 2009.

BEZERRA, J. E. F.; SILVA JUNIOR, J. F. da; LEDERMAN, I. E. Pitanga (Eugenia uniflora L.). Jaboticabal: FUNEP, 2000. 30p. (Série Frutas Nativas, 1).

BORGES, K. C.; MEDEIROS, A. C. L.; CORREIA, R. T. P. Iogurte de leite de búfala sabor Cajá (Spondias lútea L.). Caracterização físico-química e aceitação sensorial, entre indivíduos de 11 a 16 anos. Alimentos e Nutrição, Araraquara, v. 20, n. 2, p. 295-300, 2009.

BRASIL. Ministério da Agricultura, Pecuária e Abastecimento Instrução Normativa $n^{0} 46$. Aprova o regulamento técnico de produção, identidade e qualidade do leite tipo A, o regulamento técnico de identidade e qualidade de leite cru refrigerado, o regulamento técnico de identidade e qualidade de leite pasteurizado e o regulamento técnico da coleta de leite cru refrigerado e seu transporte a granel. 2011. Diário Oficial da República Federativa do Brasil, Brasília, DF, 30 dez. 2011.

BRASIL. Ministério da Agricultura, Pecuária e Abastecimento. Instrução Normativa $n^{0} 51$. Aprova e oficializa o regulamento técnico de identidade e qualidade de leite cru refrigerado.
2002. Diário Oficial da República Federativa do Brasil, Brasília, 2002.

BRASIL. Ministério da Agricultura, Pecuária e Abastecimento Instrução Normativa $n^{0} 62$. Regulamento técnico de identidade e qualidade de leites fermentados. 2007. Diário Oficial da República Federativa do Brasil, Brasília, 24 out. 2007.

BRASIL. Ministério da Agricultura, do Abastecimento e da Reforma Agrária. Resolução $\mathrm{n}^{\mathrm{o}}$ 5. Padrões de identidade e qualidade (PIQ) de leites fermentados. 2000. Diário Oficial da República Federativa do Brasil, Brasília, DF, 27 nov. 2000 .

COELHO, K. O. et al. Determinação do perfil físico-químico de amostras de leite de búfalas, por meio de analisadores automatizados. Ciência Animal Brasileira, v. 5, n. 3, p. $167-$ 170, 2004

CUNHA NETO, O. C. et al. Avaliação do iogurte natural produzido com leite de búfala contendo diferentes níveis de gordura. Ciência e Tecnologia de Alimentos, Campinas, v. 3, n. 25, p. 448-453, 2005.

DUTCOSKY, S. D. Análise Sensorial de Alimentos. $2^{\mathrm{a}}$ ed. Curitiba: Editora Universitária, 2007. 239 p.

FAVARO-TRINDADE, C. S. et al. Sensory acceptability and stability of probiotic microorganisms and vitamin $\mathrm{C}$ in fermented acerola (Malpighia emarginata DC.) ice cream. Journal of Food Science, v. 71, n. 6, p. 492495, 2006.

GUERRA, R. B.; NEVES, E. C. A.; PENA, R. S. Caracterização e processamento de leite bubalino em pó em secador por nebulização. Ciência e Tecnologia de Alimentos, Campinas, v. 25, n. 3, p. 443-447, 2005.

INSTITUTO ADOLFO LUTZ (IAL). Métodos 
físico-químicos para análise de alimentos. $4^{\mathrm{a}}$ ed. São Paulo: Instituto Adolfo Lutz, 2008. $1020 \mathrm{p}$.

LIMA, V. L. A. G.; MÉLO, E. A.; GUERRA, N. B. Correlação entre o teor de antocianinas e caracterização cromática de polpas de diferentes genótipos de aceroleira. Brazilian Journal Food of Technology, v. 10, n. 1, p. 51$55,2007$.

LOPES, F. A. Caracterização da produtividade e da qualidade do leite de búfalas na Zona da Mata Sul de Pernambuco. 2009. 45 f. Dissertação (Mestrado em Zootecnia) Universidade Federal Rural de Pernambuco, Recife, 2009.

MEDEIROS, F. C. et al. Composição centesimal de iogurtes comercializados no município de Bananeiras-PB. In: JORNADA NACIONAL DA AGROINDÚSTRIA, 2., 2007, Bananeiras. Anais... Bananeiras: UFPB, 2007. CD-ROM.

MILLER, G. L.; Use of dinitrosalicylic acid reagent for determination of reducing sugar. Analitycal Chemistry, v. 31, n. 3, p. 126128, 1959.

NEVES, N. R. P. Elaboração de iogurte de açaí (Euterpe oleracea Mart.) tipo "sundae". 2007. Trabalho de Conclusão de Curso (Graduação em Tecnologia Agroindustrial com ênfase em Alimentos) - Universidade do Estado do Pará, Redenção, 2007.

OLIVEIRA, F. M. N.; FIGUÊIREDO, R. M. F.; QUEIROZ, A. J. M. Análise comparativa de polpas de pitanga integral, formulada e em pó. Revista Brasileira de Produtos Agroindustriais, Campina Grande, v. 8, n. 1, p. 25-33, 2006.

OLIVEIRA, R. L. Composição química e perfil de ácidos graxos do leite e muçarela de búfalas alimentadas com diferentes fontes de lipídeos. Arquivo Brasileiro de Medicina
Veterinária e Zootecnia, v. 61, n. 3, p. 736744, 2009.

SANCHOTENE, M. C. C. Frutíferas nativas úteis a fauna na arborização urbana. $2^{\mathrm{a}} \mathrm{ed}$. Porto Alegre: Sagra, 1989. 304p.

SANTOS, A. F. et al. Alterações fisiológicas durante a maturação de pitanga (Eugenia uniflora L.). Proceedings of the Interamerican Society for Tropical Horticulture, Beltsville, v. 46, p. 52-54, 2003.

SECRETARIA DE AGRICULTURA E ABASTECIMENTO (SAA). Resolução SAA $\mathrm{n}^{\circ}$ 24. Normas técnicas de produção e classificação dos produtos de origem animal, atividades de fiscalização e inspeção dos produtos de origem animal. 1994. Diário Oficial do Estado de São Paulo, São Paulo, 02 ago. 1994.

SILVA, S. L. A.; FERREIRA, G. A. L.; SILVA, R. R. À procura da vitamina C. Química Nova na Escola, n. 2, 1995.

SOUZA R. M. S. R. Iogurte de leite de búfala adicionado de polpa de frutas da Amazônia: parâmetros de qualidade. 2011. 85 f. Tese (Doutorado em Higiene Sanitária e Processamento Tecnológico de Produtos de Origem Animal) - Universidade Federal Fluminense, Niterói, 2011.

STATSOFT INC. Statistica Kernel Release 7.1.: Computer program manual. Tulsa, 2006. software.

VERRUMA, M. R.; OLIVEIRA, A. J. Avaliação química e nutricional do queijo Mozzarela e iogurte de leite de búfala. Scientia Agrícola. Piracicaba, v. 50, n. 3, p. 438-443, 1993.

VERRUMA, M. R.; SALGADO J. M. Análise química do leite de búfala em comparação ao leite de vaca. Scientia Agrícola. Piracicaba, v. 51, p. 131-137, 1994. 